\title{
ON LOCAL TREES
}

L. E. WARD, JR.

1. Introduction. In the Menger classification of curves as augmented by Whyburn [12], the Peano continua are arranged in the following hierarchy:

$$
\text { (I) } \subset \text { (II) } \subset \text { (III) } \subset \text { (IV) } \subset\left(\mathrm{V}_{1}\right) \subset \cdots \subset\left(\mathrm{V}_{n}\right) \subset \cdots
$$

where I denotes the class of dendrites, II is the regular curves, III is the hereditarily locally connected continua, IV is the rational curves, and $\mathrm{V}_{n}$ is the class of continua of dimension not exceeding $n$. Now it should be remarked that, for each of these classes save the first, if a continuum possesses the defining property in the small then it is a member of the class. That is, a local regular curve is a regular curve, a local rational curve is a rational curve, and so on, but a local dendrite is not in general a dendrite. However, a local dendrite is a regular curve (but not conversely) so that between the classes I and II there is a distinct and interesting class of continua which warrants study. So far as the author is aware this class has received scant attention in the literature. In this paper we give three characterizations of local dendrites and trees. We show that a Peano continuum is a local dendrite if and only if it contains no small simple closed curves and if and only if it is an absolute neighborhood retract of dimension not exceeding unity. The third characterization is order-theoretic and is an extension of a similar theorem of the author's for trees [8].

The problem of characterizing spaces in terms of their order properties deserves further comment. In the presence of arcwise connectedness and very strong unicoherence properties the inherent order structure of connected spaces has proved to be an effective tool in characterization theorems $[2 ; 5 ; 8-11]$. However, even such a simple class of spaces as the regular curves has offered considerable difficulty and so far has not been tractable to discussion in terms of order. Two results lend encouragement to the task. Wallace [6] has shown that only the indecomposable continua have truly trivial partial order relations and the author [7] has shown that any locally connected continuum can be partially ordered in terms of its cutpoints. If, in the latter case, a way can be found to "extend" the partial order over the cyclic elements of a locally connected continuum

Received by the editors December 28, 1959.

This research was supported in part by the United States Air Force under Contract No. AF $49(638)-889$. 
then one may reasonably expect to translate special topological properties into order-theoretic terms, and vice versa.

2. Topological properties of local trees. We begin by recalling a few definitions $[8 ; 9]$. A tree is a continuum in which each two distinct points can be separated by the omission of some third point. It is well-known that a tree is locally connected and that a metric tree is a dendrite, i.e., a Peano continuum which contains no simple closed curve. A local tree is a connected space in which each point has arbitrarily small closed neighborhoods that are trees. Thus a local tree is necessarily locally connected and locally compact, a tree is a local tree, and a compact metric local tree is a regular curve. The reverse inclusions fail; for example, a simple closed curve is a local tree but not a tree, and a regular curve may possess arbitrarily small simple closed curves while a compact local tree may not.

Definition. Let $X$ be a metric space; then $X$ contains no small simple closed curves if and only if there is an $\epsilon>0$ such that diam $(S)$ $\geqq \epsilon$ for each simple closed curve $S$ contained in $X$.

Theorem 1. Let $X$ be a connected metric space. If $X$ is locally compact, locally connected, and contains no small simple closed curves, then $X$ is a local tree. Conversely, if $X$ is a compact local tree then $X$ contains no small simple closed curves.

Proof. Suppose diam $(S) \geqq \epsilon>0$ for each simple closed curve $S$ contained in $X$. Let $x \in X$ and let $N$ be a compact connected neighborhood of $x$ contained in the $\epsilon / 2$-sphere about $x$. Obviously $N$ is a tree. To prove the converse, suppose $X$ is compact and contains a sequence $S_{n}$ of simple closed curves with diam $\left(S_{n}\right)<1 / n$. Then lim sup $S_{n}$ must contain a point $x$ of $X$ and each neighborhood of $x$ contains an $S_{n}$ and hence $X$ cannot be a local tree.

Corollary. A Peano continuum is a local tree if and only if it contains no small simple closed curves.

In the next theorem we use the codimension function of Haskell Cohen [3]. Let $H^{n}(X)$ denote the Alexander-Kolmogoroff $n$-dimensional cohomology group of the compact space $X$. Then $X$ has codimension not exceeding $n$ (in symbols, $\operatorname{cd}(X) \leqq n$ ) if and only if the natural homomorphism $H^{n}(X) \rightarrow H^{n}(A)$ is onto for each closed subset $A$ of $X$. It is known that if $\operatorname{dim}(X)=1$ in the Menger-Urysohn sense, then $\operatorname{cd}(X)=1$.

THEOREM 2. If $X$ is a compact metric absolute neighborhood retract and $\operatorname{dim}(X)=1$ then $X$ contains no small simple closed curves. 
Proof. Suppose, on the other hand, that $X$ contains arbitrarily small simple closed curves. Then $X$ contains a sequence $S_{n}$ of simple closed curves and $\lim S_{n}=x$, an element of $X$. Since $\operatorname{dim}(X)=1$ we can imbed $X$ in Euclidean 3-space; since $X$ is an absolute neighborhood retract there is an open set $U$ of 3 -space and a retraction $\pi(U)$ $=X$. In particular, there is an $\epsilon>0$ such that $\bar{V} \subset U$ and $V$ is the open $\epsilon$-sphere about $x$. Moreover, for sufficiently large $n, S_{n} \subset \bar{V}$. Let $\sigma$ denote the function $\pi$ restricted to $\bar{V}$ and let $i$ and $j$ be the injective functions $i: S_{n} \rightarrow \bar{V}, j: S_{n} \rightarrow X$. Then $j^{*}=(\sigma i)^{*}=i^{*} \sigma^{*}$, where $j^{*}: H^{1}(X)$ $\rightarrow H^{1}\left(S_{n}\right), i^{*}: H^{1}(\bar{V}) \rightarrow H^{1}\left(S_{n}\right)$, and $\sigma^{*}: H^{1}(X) \rightarrow H^{1}(\bar{V})$ are the induced natural homomorphisms. Since $\operatorname{cd}(X)=1, j^{*}$ is onto and hence $i^{*}$ is onto. But, since $H^{1}(\bar{V})=0$ and $H^{1}\left(S_{n}\right) \neq 0$, this is impossible.

THEOREM 3. Let $X$ be a metric space. If $X$ is a local tree then $X$ is an absolute neighborhood retract. Conversely, if $X$ is a compact connected absolute neighborhood retract and $\operatorname{dim}(X)=1$, then $X$ is a local tree.

Proof. Clearly, if $U$ is a connected open subset of $X$ such that $\bar{U}$ is a tree, then $U$ is an absolute neighborhood retract. Further, a locally separable, connected metric space is separable [1], so that $X$ is separable. Hence, by a theorem of Hanner [4], $X$ is an absolute neighborhood retract. The converse follows from Theorems 1 and 2.

Corollary. A Peano continuum is a local tree if and only if it is an absolute neighborhood retract whose dimension does not exceed unity.

3. Order properties of local trees. Let $X$ be a connected space and $e \in X$. Define $x \leqq y$ for elements $x$ and $y$ of $X$ if and only if $x=e$ or $x=y$ or $x$ separates $e$ and $y$ in $X$. It is easily seen that $\leqq$ is a partial order on the elements of $X$ and, if $X$ is a locally connected continuum, the graph of $\leqq$ is a closed subset of $X \times X$ [7]. The relation $\leqq$ is termed the cutpoint ordering of $X$ with minimal element $e$ and has been used [8] to characterize the trees. For its applicability to local trees, we state that result here. The sets $L(x)=\{y: y \leqq x\}$ and $M(x)$ $=\{y: x \leqq y\}$ are, respectively, the sets of predecessors and successors of $x$, relative to the partial order $\leqq$. We write $x<y$ when $x \leqq y$ and $x \neq y$. A chain of a partially ordered set is a subset which is simple with respect to the partial order.

THeOREM 4. Let $X$ be a compact Hausdorff space. A necessary and sufficient condition that $X$ be a tree is that $X$ admit a partial order $\leqq$ such that

(i) $\leqq$ has a closed graph,

(ii) if $x<y$ then there exists $z \in X$ such that $x<z$ and $z<y$, 
(iii) for each $x$ and $y$ in $X$, the set $L(x) \cap L(y)$ is a nonempty chain,

(iv) for each $x \in X, M(x)-x$ is an open set.

From (iii) and the fact [7] that any compact partially ordered space has a minimal element, it is seen that the order of Theorem 4 has a unique minimal element. The cutpoint ordering with that element as minimal is precisely the (unique) order with properties (i)-(iv).

Theorem 5. Let $X$ be a compact Hausdorff space. A necessary and sufficient condition that $X$ be a local tree is that $X$ admit a partial order satisfying (i) and (ii) of Theorem 4 and also contain a finite set $E=\left\{e_{1}, \cdots, e_{N}\right\}$ such that

(iii') $M\left(e_{1}\right)=X$,

(iv') for each $j=1, \cdots, N$, the set

$$
A_{j}=\left(M\left(e_{j}\right)-e_{j}\right)-\bigcup_{k>j}\left\{M\left(e_{k}\right)\right\}
$$

is open and $\bar{A}_{j}$ is a tree with the cutpoint ordering with $e_{j}$ as minimal element.

Proof. If $X$ is a local tree then $X$ can be covered by a finite family $\mathcal{u}$ of connected open sets such that, for each $U \in \mathcal{U}, \bar{U}$ is a tree and $\bar{U}-U$ is finite. If $\mathfrak{u}$ has only one member then $X$ is a tree and the desired partial order is inferred from Theorem 4 . If $\mathfrak{u}$ contains more than one member, let $V_{1} \in \mathcal{U}, e_{1} \in V_{1}$ and $e_{2} \in \bar{V}_{1}-V_{1}$. Let $U_{2} \in \mathcal{U}$ such that $e_{2} \in U_{2}$ and let $V_{2}$ be the union of all components of $U_{2}-\bar{V}_{1}$ whose closures contain $e_{2}$. Then $V_{2}$ is an open subset of $X$ whose closure is connected, contained in $\bar{U}_{2}$, and hence is a tree. Moreover, $\bar{V}_{1} \cup \bar{V}_{2}$ has a finite boundary. Assuming $V_{n}$ has been selected and that $\bar{V}_{1} \cup \ldots \cup \bar{V}_{n} \neq X$, let $e_{n+1}$ be an element of the boundary of $\bar{V}_{1} \cup \ldots \cup \bar{V}_{n}$. Let $U_{n+1} \in \mathcal{U}$ such that $e_{n+1} \in U_{n+1}$ and let $V_{n+1}$ be the union of all components of $U_{n+1}-\left(\bar{V}_{1} \cup \cdots \cup \bar{V}_{n}\right)$ whose closures contain $e_{n+1}$.

For each $n>1, e_{n}$ lies in the boundary of $\bar{V}_{1} \cup \ldots \cup \bar{V}_{n-1}$, hence also in the boundary of $\bar{U}_{1} \cup \ldots \cup \bar{U}_{n-1}$, and therefore in the boundary of some $\bar{U}_{k}$. Since $\mathfrak{U}$ is finite and each $U \in \mathcal{U}$ has finite boundary, only a finite number of $\bar{V}_{n}$ can be formed. Further, $X$ is covered by such sets and hence there is an integer $N$ such that $X=\bar{V}_{1} \cup \ldots$ $\cup \bar{V}_{N}$.

For each $n=1,2, \cdots, N$, give $\bar{V}_{n}$ the cutpoint ordering with $e_{n}$ as minimal element, and let $E=\left\{e_{1}, \cdots, e_{N}\right\}$. It remains to extend the partial order over $X$ by defining $x \leqq y$ when $x$ and $y$ do not belong to the same $\bar{V}_{n}$. Suppose $x \in \bar{V}_{n}$ and $y \in \bar{V}_{m}$ implies $n \neq m$. Then $x \leqq y$ only if $n<m$ and there is a sequence $e_{n_{1}}, e_{n_{2}}, \cdots, e_{n_{p}}=e_{m}$ of elements 
of $E$ such that $e_{n_{1}} \in \bar{V}_{n}-e_{n}, e_{n_{2}} \in \bar{V}_{n_{1}}-e_{n_{1}}$, and so forth, and $x \leqq e_{n_{1}}$ in the cutpoint ordering of $\bar{V}_{n}$. It is readily verified that the extended relation $\leqq$ is a partial order satisfying (i), (ii) and (iii'). Further $A_{1}=V_{1}-e_{1}$ and $A_{j}=V_{j}$ for $j>1$, so that (iv') is clear.

To establish the converse, suppose $X$ admits a partial order satisfying (i), (ii), (iii'), and (iv'). It follows from (ii) and (iii') that $X$ is connected (Theorem 4 of [7]). If $x \in X-E$, then there is a greatest $j$ such that $x \in M\left(e_{j}\right)$ and hence $x \in A_{j}$, that is, $X$ is a local tree at $x$. If $x=e_{1}$ then $A_{1} \cup\left\{e_{1}\right\}$ is the desired neighborhood of $x$. If $x=e_{j}$, $j>1$, then $x$ is an endpoint of $\bar{A}_{j}$ and also of some $\bar{A}_{i}$ where $x$ is maximal in the latter set. The set $\bar{A}_{i} \cap \bar{A}_{j}$ is finite and both $\bar{A}_{i}$ and $\bar{A}_{j}$ are trees. Hence $A_{i} \cup A_{j} \cup\{x\}$ is a neighborhood of $x$, every subcontinuum of which is a tree, so that $X$ is a local tree at $x$.

\section{REFERENCES}

1. Paul Alexandroff, Über die Metrization der im kleinen kompakten topologischen Raume, Math. Ann. vol. 92 (1924) pp. 294-301.

2. L. W. Anderson and L. E. Ward, Jr., One dimensional topological semi-lattices, to appear, Illinois J. Math.

3. Haskell Cohen, $A$ cohomological definition of dimension for locally compact Hausdorff spaces, Duke Math. J. vol. 21 (1954) pp. 209-224.

4. Olof Hanner, Some theorems on absolute neighborhood retracts, Ark. Math. vol. 1 (1951) pp. 389-408.

5. R. J. Koch and I. S. Krule, Weak cutpoint ordering in hereditarily unicoherent continua, Proc. Amer. Math. Soc. vol. 11 (1960) pp. 679-681.

6. A. D. Wallace, Partial order and indecomposability, Proc. Amer. Math. Soc. vol. 5 (1954) pp. 780-781.

7. L. E. Ward, Jr., Partially ordered topological spaces, Proc. Amer. Math. Soc. vol. 5 (1954) pp. 144-161.

8. - A note on dendrites and trees, Proc. Amer. Math. Soc. vol. 5 (1954) pp. 992-994.

9. - On dendritic sets, Duke Math. J. vol. 25 (1958) pp. 505-514.

10. — A fixed point theorem for multi-valued functions, Pacific J. Math. vol. 8 (1958) pp. 921-927.

11. - A fixed point theorem for chained spaces, Pacific J. Math. vol. 9 (1959) pp. $1273-1278$.

12. G. T. Whyburn, Analytic topology, New York, 1942.

UNIVERSITY OF OREGON 